\title{
Introduction: Cell adhesion and extracellular matrix molecules in synaptic plasticity
}

This issue of Neuron Glia Biology contains a special collection of original research papers and reviews on the role of cell adhesion and extracellular matrix (ECM) molecules in synaptic plasticity. These molecules are crucially required for building and maintaining synaptic structure during brain development and there is increasing evidence that they also play important roles in modulating distinct aspects of synaptic plasticity in mature nervous system.

Neuronal cell adhesion molecule (NCAM), the member of immunoglobulin family, was the first vertebrate molecule to be identified and characterized as a cell adhesion molecule. NCAM is known as the major carrier of polyanionic carbohydrate polysialic acid (PSA-NCAM) that is highly expressed during brain development, contributing to the regulation of cell shape, growth or migration. Also in adult brain, PSA-NCAM expression does persist in structures that display a high degree of plasticity, such as the hippocampus, and is involved in activity-induced synaptic plasticity. In their research manuscript Rodríguez et al. (2009) demonstrate that high-frequency stimulation of medial and lateral perforant path in the dentate gyrus results in NMDA-dependent homosynaptic long-term potentiation (LTP) and heterosynaptic long-term depression (LTD) as recorded electrophysiologically in rats in vivo. This stimulation also induces increase in PSA-NCAM immunoreactivity that persists up to $24 \mathrm{~h}$ after stimulation. At the ultrustructural level, electron microscopy shows decreased PSA-NCAM dendritic labeling after heterosynaptic LTD and the sub-cellular relocation of PSA-NCAM to the spines after homosynaptic LTP, that are independent of NMDA receptor activation. These findings suggest that strong activation of the granule cells in dentate gyrus up-regulates PSA-NCAM synthesis in the cell body, with subsequent transport to the dendrite and incorporation into activated synapses, representing NMDA-independent plastic processes that may act synergistically with LTP/LTD mechanisms.

The review by Dityatev et al. (2009) summarizes the roles of cell adhesion molecules of the immunoglobulin superfamily (Ig-CAMs) and semaphorins (some of which also contain Ig-like domains) in regulation of synaptic transmission and plasticity at multiple subtypes of excitatory synapses in the hippocampus. The Ig-CAMs discussed in this review, including NCAM, L1, CHL1, neuroplastin, Thy-1, contactin-1 and semaphorins, belong to distinct subfamilies. Interestingly, among Ig-CAMs, only NCAM proved to be important for all tested forms of hippocampal plasticity. The emerging mechanisms by which adhesive Ig-CAMs contribute to synaptic plasticity involve regulation of activities of NMDA receptors and L-type $\mathrm{Ca}^{2+}$ channels, signaling via mitogenactivated protein kinase $\mathrm{p}_{3} 8$, changes in GABAergic inhibition and motility of synaptic elements. Regarding repellent molecules, available data for semaphorins demonstrate their activity-dependent regulation in normal and pathological conditions, synaptic localization of their receptors and their potential to elevate or inhibit synaptic transmission either directly or indirectly.

Integrin receptors represent another class of recognition molecules that mediate adhesion of the cell to the ECM and thereby regulate cell motility, proliferation, differentiation and apoptosis, as well as stabilize activity-induced increases in synaptic strength and excitability. In this special issue Cingolani and Goda (2009) further examine the role of $\beta_{3}$ in homeostatic plasticity in organotypic slices, and ask if homeostatic plasticity may function differently between dissociated cultured neurons and organotypic slices. Using electrophysiological recordings in dissociated culture and organotypic slices prepared from $\beta_{3}$ integrin knockout mice, several forms of homeostatic synaptic regulation are investigated. Results of this study demonstrate that $\beta_{3}$ integrin is specifically required for a postsynaptic form of synaptic homeostasis called synaptic scaling in both dissociated cultures and organotypic slices. Another form of synaptic homeostasis that involves changes in presynaptic quantal content occurs independently of $\beta_{3}$ integrin. This study shows that homeostatic synaptic plasticity induced by chronic TTX treatment is partially mechanistically different between primary cultures and organotypic slice cultures, indicating that mechanisms for homeostatic regulation in native brain may be distinct from those in neuronal culture preparation.

Receptor tyrosine kinases (RTKs) are cell surface molecules implicated in a variety of neuronal functions, including neuronal survival, axon and dendrite outgrowth, and synapse development. Two families of RTKs, ErbBs and Ephs, exhibit similar characteristics in their bi-directional signaling transduction. The forward and reverse signaling of ErbBs and Ephs have been implicated in various aspects of synapse development, including dendritic spine morphogenesis, synapse formation, maintenance and plasticity. Chen et al. (2009) in their review discuss the latest advances in the functions of ErbBs and Ephs, as well as their ligands neuregulins (NRG) and ephrins, at the synapse, including dendritic spine morphogenesis, synapse formation and maturation. Regulated trans-synaptic interaction between RTKs receptors and their ligands is essential for synaptic transmission and plasticity. In addition to signaling at excitatory glutamatergic and inhibitory GABAergic synapses, communication between neuron and glia is increasingly implicated in the control of synaptic functions and is mediated by NRG/ErbB and ephrin/Eph signaling. Implications of signaling events, mediated by RTKs and their cognate ligands, in human diseases is also discussed.

The importance of proteolytic activity regulation at or near the synapses, or perisynaptic proteolysis, as one of the mechanisms underlying synaptic plasticity is discussed in the review by Lee et al. (2009). It has been demonstrated that perisynaptic release and/or activation of various proteases is dependent on neuronal activity. This is followed by cleavage 
of selected ECM and cell adhesion molecules, leading to the alterations in structural and adhesive properties at the synapses, as well as changes in signaling and downstream effects, that manifested as long-term changes in synaptic transmission. In the first part of the manuscript authors describe the importance of proteases in neurotransmission and synaptic plasticity, reviewing a literature on metalloproteases, ADAM family proteases, serine proteases (tissue plasminogen activator/plasmin system and neuropsin) and aspartic proteases. Secondly, studies of matrix and cell adhesion molecules that have established roles in neuronal plasticity have highlighted how proteolytic processing plays an important role in regulating their function. As example of how proteolitic processing leads to plasticity-related functional authors described the processing of $\mathrm{N}$-cadherin, NCAM, $\mathrm{L}_{1}$ and reelin.

The ECM molecules, derived from both neurons and glial cells, are secreted and accumulate in the extracellular space to regulate maturation of synapses and shape synaptic plasticity. Dityatev and Fellin (2009), in their review, discuss the contribution of ECM molecules, including chondroitin sulfate and heparin sulfate proteoglycans, tenascins, reelin and proteases to different forms of plasticity. Modulation of developmental plasticity in the cortex, LTP and LTD in the hippocampus, homeostatic scaling of synaptic transmission and metaplasticity by these ECM molecules involves different mechanisms. Mutation in some ECM molecules leads to alterations in the development of GABAergic and glutamatergic transmission, suggesting that the excitation-inhibition unbalance might be a mechanism through which ECM regulates different forms of plasticity and epileptogenesis. Indeed, both neuronal stimulation leading to LTP and epileptic seizures results in changes of ECM components expression. ECM molecules are also engaged in different aspects of epileptogenesis, such as dysregulation of excitatory and inhibitory neurotransmission, sprouting of mossy fibers, granule cell dispersion and gliosis. Authors proposed that the restraining of ECM modification might be effective therapeutic interventions to antagonize the progression of epileptogenesis.

Many neurons and their synapses are enwrapped in a brain-specific form of ECM, the so-called perineuronal net (PNN), which forms late in the postnatal development around the time when synaptic contacts are stabilized. PNN is composed of glycoproteins and proteoglycans of glial and neuronal origin. Frischknecht and Seidenbecher (2009) review findings on hyaluronic acid (HA), also termed hyaluronan, a major organizing component of PNN and its role in synapse development and function. Biochemistry of hyaluronan, HA synthesis and degradation, neuronal expression of HA enzymes and HA-binding receptors are well presented in the beginning of this review. Then authors review available data on hyaluronan-containing PNN formation and composition during the brain development, degradation of HA-ECM by exogenous glycosidases and perysinaptic localization of PNN components. Retrospective and recent findings on hyaluronan and HA-associated ECM components in synaptic plasticity are addressed in the second part of the review. Authors also discuss the role of HA in models of regenerative plasticity and outline future investigations of HA via an endogenous regulation of hyaluronan-based ECM.

Recent data from several groups indicate that reelin, a large glycoprotein associated with the ECM, is essential for normal brain development and successful formation of certain forms of long-term memory. Research is only now beginning to understand the postnatal role of reelin in the cortex and hippocampus, but almost nothing is known about reelin's role in the cerebellum. Combining immunocytochemical and pharmacological approaches Sinagra et al. (2009) characterized the phenotype and temporal profile of reelin expression in dissociated culture of cerebellar neurons. They showed for the first time that a homogeneous population of glutamatergic neurons in the cerebellum can express several components of the reelin signaling pathway, including reelin and it receptors apolipoprotein E receptor 2 and very lowdensity lipoprotein receptor. Authors demonstrated that all reelin immunoreactive cerebellar granule cells synthesize and secrete reelin in vitro. The role reelin is playing in the cerebellar synaptic plasticity remains to be determined.

The review by Rogers and Weeber (2009) highlighted a series of studies investigating apolipoprotein $\mathrm{E}$ (apoE) and reelin, two ligands of low-density-lipoprotein receptors. Authors reviewed the importance of these ligands for normal brain development and synapse maturation. Notably, both glial-derived apoE and interneuron-derived reelin are involved in modulating hippocampal synaptic plasticity. Acute exogenous reelin application leads to enhanced hippocampal LTP, mediated by increasing $\mathrm{Ca}^{2+}$ and NMDA receptor conductivity, whereas long-term exposure affects AMPA receptor-mediated synaptic responses and leads to the insertion of AMPA receptors. Acute application of specific apoE isoforms have different effects on signal transduction pathways involved in LTP induction (increasing or decreasing LTP). Unlike reelin, specific apoE isoforms can alter synaptic plasticity in a subregion-specific manner. The authors suggest that reelin and apoE signaling can have a dramatic impact on synaptic strength and plasticity in normal brain, and propose investigating the implication of apoE and reelin misregulation in the memory dysfunction component of neurodegenerative and neuropsychiatric disorders.

In summary, the available experimental evidence indicates that cell adhesion molecules and ECM components affect the function of synapses at multiple levels, from regulating morphology to modulating the function of synaptic channels and receptors and regulating synaptic plasticity. The interplay between cell adhesion and ECM molecules at certain types of cells and synapses, both during development and in the mature central nervous system, could be important for understanding synaptic transmission mechanism. Further roles of cell-cell and cell-matrix interactions, as well as dynamic remodeling of ECM in normal and pathological brain are just becoming apparent and are expected to be growing area of research.

\section{REFERENCES}

Chen Y., Fu A.K.Y. and Ip N.Y. (2009) Bidirectional signaling of ErbB and Eph receptors at synapses. Neuron Glia Biology 4, 211-221.

Cingolani L.A. and Goda Y. (2009) Differential involvement of $\beta_{3}$ integrin in pre- and postsynaptic forms of adaptation to chronic activity deprivation. Neuron Glia Biology 4, 179-187.

Dityatev A., Bukalo O. and Schachner M. (2009) Modulation of synaptic transmission and plasticity by cell adhesion and repulsion molecules. Neuron Glia Biology 4, 197-209. 
Dityatev A. and Fellin T. (2009) Extracellular matrix in plasticity and epileptogenesis. Neuron Glia Biology 4, 235-247.

Frischknecht R. and Seidenbecher C. (2009) The crosstalk of hyaluronan-based ECM and synapses. Neuron Glia Biology 4, 249-257.

Lee T.W., Tsang V.W.K. and Birch N.P. (2009) Synaptic plasticity-associated proteases and protease inhibitors in the brain linked to the processing of extracellular matrix and cell adhesion molecules. Neuron Glia Biology 4, 223-234.

Rodríguez J.J., Dallérac G.M., Tabuchi M., Davies H.A., Colyer F.M., Stewart M.G. et al. (2009) N-methyl-D-aspartate receptor independent changes in expression of polysialic acid-neural cell adhesion molecule despite blockade of homosynaptic long-term potentiation and heterosynaptic long-term depression in the awake freely behaving rat dentate gyrus. Neuron Glia Biology 4, 169-178.

Rogers J.T. and Weeber E.J. (2009) Reelin and apoE actions on signal transduction, synaptic function and memory formation. Neuron Glia Biology 4, 259-270.

Sinagra M., Gonzalez Campo C., Verrier D., Moustié O., Manzini O.J. and Chavis P. (2009) Glutamatergic cerebellar granule neurons synthesize and secrete reelin in vitro. Neuron Glia Biology 4, 189-196.

Olena Bukalo

Special Edition Associate Editor,

Neuron Glia Biology 